\title{
Die NAIRU - eine post-keynesianische Interpretation
}

\author{
Eckhard Hein*
}

\begin{abstract}
In this paper the New Keynesian long run equilibrium view of a non-accelerating-inflation-rate-of-unemployment (NAIRU) determined by structural characteristics of the labour market, wage bargaining institutions and social benefit systems is challenged by a Post-Keynesian view. In a Post-Keynesian model the NAIRU can only be interpreted as a short run barrier to employment arising from inflation fuelled by distribution conflict, which is enforced by monetary policies. In the long run, the development of the NAIRU follows actual unemployment and hence effective demand. Different adjustment channels are identified. It is also shown that effective coordination of wage bargaining is a better means to stabilise inflation rates and employment than monetary policies.
\end{abstract}

\section{Einführung}

Das Konzept einer inflationsstabilen Arbeitslosenquote, einer Non-Accelerating Inflation Rate of Unemployment (NAIRU), gehört mittlerweile zum allgemein akzeptierten Kern des in den USA dominierenden neu-keynesianischen makroökonomischen Mainstreams (vgl. Ball/Mankiw 2002, Mankiw 200I, Stiglitz 1997). Auch den neu-keynesia-

* Wirtschafts- und Sozialwissenschaftliches Institut in der Hans-Böckler-Stiftung, Düsseldorf. Ich danke Achim Truger, Thorsten Schulten, Jan Priewe, Torsten Niechoj, Hansjörg Herr, Arne Heise und Trevor Evans für klärende Diskussionen, Barbara Schnieders für die Durchsicht des Manuskriptes sowie zwei anonymen GutachterInnen für hilfreiche Kommentare. Verbliebene Irrtümer gehen selbstverständlich zu meinen Lasten.

Korrespondenzanschrift:

Dr. Eckhard Hein, Wirtschafts- und Sozialwissenschaftliches Institut in der Hans-Böckler-Stiftung, Hans-Böckler-Str. 39, 40476 Düsseldorf, E-mail: eckhard-hein@boeckler.de

Ersteinreichung des Artikels am 13.6.2003, endgültig akzeptiert am 12.8.2003

(C) InTERvention. Zeitschrift für Ökonomie, Jg. 1 (2004), H. 1, S. 43-66 
nisch inspirierten neuen "Konsensmodellen« für die Analyse der makroökonomischen Wirkungen der Geldpolitik liegt implizit das Konzept einer NAIRU zugrunde (vgl. Arestis/Sawyer 2003, Clarida/Gali/Gertler 1999, Meyer 200I). ${ }^{1}$ Aufgrund der nun, im Gegensatz zur Neoklassischen Synthese, »mikrofundierten« nominalen Rigiditäten wird in diesem neuen Mainstream wieder die Gültigkeit der Phillips-Kurve und damit des Trade-off von Inflation und Arbeitslosigkeit für die kurze Frist akzeptiert, die von den Protagonisten der in den I980er-Jahren dominierenden neu-klassischen Makroökonomie noch verworfen worden war (vgl. Heine/Herr 2003). Von der Geldpolitik beeinflusste nominale Variablen haben daher in den neu-keynesianischen Modellen, genauso wie in den alten Modellen der Neoklassischen Synthese und des Monetarismus, kurzfristig auch reale Wirkungen auf Produktion und Beschäftigung, weil Preise und Nominallöhne nicht unmittelbar auf exogene Schocks reagieren. ${ }^{2}$ Eine aktive und interventionistische Geld- und Fiskalpolitik ist in der kurzen Frist daher immer dann angezeigt, wenn die Ökonomie in eine Rezession zu gleiten droht, da die Marktanpassungsprozesse an das langfristige Gleichgewicht aufgrund von Preisrigiditäten nur sehr langsam funktionieren.

In der langen Frist wird die Arbeitslosenquote jedoch in den neu-keynesianischen Modellen durch die NAIRU bestimmt, die selbst wiederum von Strukturfaktoren des Arbeitsmarktes, der Lohnverhandlungen und der sozialen Sicherungssysteme abhängt. Die NAIRU ist dabei nicht mit Markträumung auf dem Arbeitsmarkt gleichzusetzen. Sie beschreibt vielmehr die Arbeitslosenquote, bei der die Verteilungsansprüche von ArbeiterInnen und Unternehmen auf imperfekten Arbeits- und Gütermärkten keine Veränderung der Inflationsrate nach oben oder nach unten auslösen. Zur theoretischen Bestimmung dieser Arbeitslosenquote sind verschiedene Modelle präsentiert worden: Lohnverhandlungsmodelle, in denen Reallohnhöhe und Beschäftigung von der Verhandlungsstärke von Gewerkschaften und Unternehmen abhängen, Effizienzlohnmodelle, in denen Reallöhne einen positiven Einfluss auf die Arbeitsproduktivität ausüben (Shirkingoder Fairness-Modelle), oder Modelle, in denen der durch Lohnersatzleistungen mitbestimmte Reservationslohn den minimalen Reallohn bestimmt (vgl. Blanchard/Katz 1997). Die NAIRU ist in diesen Modellen im Zeitverlauf nicht notwendig konstant,

1 Diese Modelle bestehen in ihrer einfachsten Form aus drei Gleichungen: einer aggregierten Nachfragefunktion, die aus den Optimierungskalkülen von Haushalten und Unternehmen abgeleitet wird und das Output-gap - die Differenz zwischen Produktionskapazität und tatsächlichem Output - als inverse Funktion des Realzinses darstellt, einer Phillips-Kurve, in der die Inflationsrate positiv vom Output-gap abhängt, und einer Reaktionsfunktion der Zentralbank, die den von ihr gesetzten Nominalzinssatz vom gleichgewichtigen Realzinssatz, vom Output-gap und von der Abweichung der Inflationsrate vom Inflationsziel abhängig macht (Taylor-Regel). In dem Modell spielen Erwartungen eine explizite Rolle. Das Modell weist kurzfristige Preisrigiditäten auf, langfristig hingegen sind die Preise vollkommen flexibel.

2 Als Ursachen für Preisrigiditäten werden hierbei häufig die Vermeidung von »Menükosten« einer Preisänderung auf unvollständigen Gütermärkten, als Gründe für Nominallohnrigiditäten entsprechend die Vermeidung von Verhandlungs- und Informationskosten durch längerfristige Lohnkontrakte auf Arbeitsmärkten angeführt. Vgl. z.B. Snowdon/Vane/Wynarczyk (I994: 286 ff.) zu einem Überblick über neu-keynesianische Modelle. 
sondern steigt immer dann, wenn z.B. die Flexibilität des Arbeitsmarktes abnimmt, die Verhandlungsstärke der Gewerkschaften zunimmt oder die Höhe von Lohnersatzleistungen oder des Minimum-Lohns ansteigt.

Wird für die kurze Frist noch ein Einfluss der effektiven Nachfrage auf Produktion und Beschäftigung zugestanden, so wird ein solcher Einfluss auf die langfristig gültige NAIRU in der Mehrzahl der Modelle negiert (vgl. Sawyer 20oI). Langfristig ist daher die Geldpolitik in diesen neu-keynesianischen Modellen wieder neutral (vgl. Hein 2003). Damit sich die durch die effektive Güternachfrage bestimmte Arbeitslosenquote an die durch die Angebotsbedingungen gegebene NAIRU anpasst, muss implizit oder explizit entweder die langfristige Wirksamkeit von Realkasseneffekten (Keynes- und/oder PigouEffekt) unterstellt werden, wie z.B. bei Carlin/Soskice (I990), ${ }^{3}$ oder aber es muss von symmetrischen Eingriffen und Wirkungen der Geldpolitik zwecks Verhinderung von akzelerierender Inflation oder Disinflation bzw. Deflation ausgegangen werden, wie in den eingangs erwähnten jüngeren Modellen der neu-keynesianischen Makroökonomik und den neuen »Konsensmodellen«.

Erfolgt bei dauerhafter Unterbeschäftigung und trägen Preisreaktionen, die damit die Realkasseneffekte verzögern bzw. verhindern, kein stabilisierender Eingriff der Geldpolitik (oder der Fiskalpolitik), so gestehen jedoch einige neu-keynesianische AutorInnen, insbesondere bei der Analyse der seit Beginn der I970er-Jahre langfristig gestiegenen Arbeitslosigkeit in der Europäischen Union, durchaus zu, dass die effektive Güternachfrage auch einen langfristigen Einfluss auf die NAIRU haben kann, indem ein Nachfrageschock das Hysterese-Phänomen begünstigt (vgl. Carlin/Soskice I990: 440 ff., Blanchard/Katz I997, Layard/Nickell/Jackman I99I: 256 ff., Mankiw 200I). Die NAIRU kann demnach vom Entwicklungspfad der tatsächlichen Arbeitslosigkeit abhängen, wenn z.B. Teile der Arbeitslosen bei steigender Arbeitslosigkeit und zunehmender Langzeitarbeitslosigkeit wesentliche Qualifikationen verlieren, ihre Suchaktivitäten einschränken, von den Unternehmen nicht mehr als ernsthafte BewerberInnen um einen Arbeitsplatz angesehen werden, damit keine effektive Konkurrenz auf dem Arbeitsmarkt mehr darstellen, das Insider-Outsider-Problem verstärken und die Verhandlungsmacht der Gewerkschaften erhöhen.

Obwohl in einigen neu-keynesianischen Ansätzen eine langfristige Verschiebung der NAIRU durch die Entwicklung der tatsächlichen, durch die effektive Nachfrage auf Gütermärkten bestimmten Arbeitslosigkeit durchaus zugestanden wird, bleibt die Behebung struktureller Verwerfungen am Arbeitsmarkt im Rahmen dieses Ansatzes doch das zentrale Instrument zur Bekämpfung hoher Arbeitslosigkeit (vgl. z.B. Snowdon/Vane/ Wynarczyk 1994: 325 ff., Franz 200I, Jerger 2003). Dies bedeutet zum einen, die Macht von Gewerkschaften und Insidern (»ArbeitsplatzbesitzerInnen«) zu reduzieren. Hierzu

3 Carlin/Soskice (I990: I59 ff.) z.B. bestimmen den tatsächlichen, durch die Güternachfrage bestimmten Beschäftigungsgrad in ihrem NAIRU-Modell durch einen IS-LM-Teil, in dem eine exogen gesetzte nominale Geldmenge die Anpassung der tatsächlichen Arbeitslosenquote an die NAIRU über den sog. "Keynes-Effekt« ermöglicht. 
zählen politische Maßnahmen zum Abbau von Kündigungsschutz, um Einstellungsund Entlassungskosten zu reduzieren, sowie zur Einschränkung der Arbeitskampfmöglichkeiten. Zum anderen sollen die »Marktchancen« der Outsider erhöht werden, indem deren Humankapital durch Qualifizierungsmaßnahmen aufgewertet und die Mobilität von Arbeitskräften gefördert wird. Die Orientierung von Lohnersatzleistungen an der Arbeitsbereitschaft und -fähigkeit soll zudem den Arbeitsangebotsdruck erhöhen. Höhere Lohnflexibilitäten durch Bindung von Lohnbestandteilen an die Unternehmensgewinne sollen eine höhere Reallohnflexibilität ermöglichen.

Diese hier knapp skizzierte neu-keynesianische NAIRU-Konzeption ist allerdings mit erheblichen Problemen verbunden. So ist für die NAIRU als Orientierungsgröße der Wirtschaftspolitik erforderlich, dass diese Größe empirisch präzise gemessen werden kann und dass sie im Zeitverlauf hinreichend stabil ist bzw. ihre Veränderung eindeutig erklärt werden kann. ${ }^{4}$ Dieses ist aber offensichtlich nicht der Fall. Staiger/Stock/Watson (1997) ermitteln z.B. für ihre Schätzung der US-NAIRU in der Mitte der I990er-Jahre ein 95\%Konfidenzintervall von 4,3\% bis 7,3\%. Ball/Mankiw (200I), Blanchard/Katz (1997), Gordon (1997), Jerger (2003), Franz (200I) und Stiglitz (1997) stellen in ihren Arbeiten fest, dass die NAIRU sich sowohl in den USA als auch in Europa und Deutschland im Zeitverlauf verändert hat, ohne dass jedoch Übereinstimmung über die entscheidenden Determinanten dieser Veränderungen besteht. Blanchard/Katz (I997: 52) konstatieren daher leicht resignierend: "Economists are a long way from having a good quantitative understanding of the determinants of the natural rate, either across time or across countries."

Auf die empirischen Fragen soll im vorliegenden Beitrag jedoch nicht weiter eingegangen werden, da hinter den Problemen der eindeutigen Messung der NAIRU und der Identifikation von Veränderungsdeterminanten ein sehr viel grundlegenderes theoretisches Problem vermutet werden kann. Dieses besteht darin, dass die Rolle der effektiven Nachfrage für die Arbeitslosigkeit in den neu-keynesianischen Modellen nur auf die kurze Frist beschränkt bleibt, ohne dass jedoch ein überzeugender Anpassungsmechanismus der durch die effektive Güternachfrage bestimmten tatsächlichen Arbeitslosenquote der kurzen Frist an die durch die Angebotsbedingungen bestimmte NAIRU der langen Frist benannt werden kann. Der in einigen älteren neu-keynesianischen Modellen angenommene Realkasseneffekt kann nämlich für eine Geldwirtschaft mit endogenem, über Gläubiger-Schuldner-Verhältnisse entstehenden Geld und unsicheren nominalen Profiterwartungen als Triebkraft von Produktion und Investition genauso wenig überzeugen wie die Unterstellung einer symmetrischen Wirkung der Geldpolitik in den jüngeren Ansätzen und den neuen »Konsensmodellen« (vgl. Sawyer 20oI). In einer Geldwirtschaft erscheint daher die Rolle der Geldpolitik und der Lohnverhandlungssysteme in einem anderen Licht als in den neu-keynesianischen Modellen mit der dort vorherrschenden langfristigen Gültigkeit von Sayschem Gesetz und klassischer Dichotomie von nominaler und realer Sphäre.

4 Diese Anforderung wird dann etwas eingeschränkt, wenn wirtschaftspolitisch verursachte Abweichungen von der NAIRU mit geringen Kosten revidiert werden können (vgl. Stiglitz 1997). 
Anders als in Galbraith (1997) wird in der vorliegenden Arbeit aus den empirischen und theoretischen Unzulänglichkeiten jedoch nicht geschlossen, dass das Konzept einer NAIRU komplett verworfen werden sollte. In den folgenden Abschnitten wird vielmehr der Versuch unternommen, eine Neu-Interpretation der NAIRU aus post-keynesianischer Perspektive zu liefern. Es wird sich zeigen, dass dabei durchaus an das bereits in einigen neu-keynesianischen Modellen thematisierte Hysterese-Phänomen angeknüpft werden kann. Im zweiten Abschnitt werden kurz die Grundlagen eines post-keynesianischen Modells für eine Geldwirtschaft rekapituliert. Im dritten Abschnitt wird dann die Interaktion von Geld- und Lohnpolitik bei gesamtwirtschaftlich unkoordinierten Lohnverhandlungen analysiert und es wird eine post-keynesianische Interpretation der NAIRU als geldpolitisch erzwungene, nur kurzfristig wirksame Inflationsbarriere für einen Abbau von Arbeitslosigkeit präsentiert, die jedoch nicht als Gravitationszentrum für die tatsächliche Arbeitslosenquote wirkt, sondern dieser vielmehr langfristig folgt. Im vierten Abschnitt wird dann auf den Einfluss der Lohnverhandlungssysteme auf die NAIRU eingegangen und es wird argumentiert, dass gesamtwirtschaftlich effektiv koordinierte Lohnbildungssysteme in der Lage sind, die NAIRU zu reduzieren und die effektive Nachfrage und Beschäftigung zu stabilisieren. Die wesentlichen Ergebnisse der hier angestellten Überlegungen werden im fünften Abschnitt kurz zusammengefasst.

\section{Grundlagen eines post-keynesianischen Modells}

Bevor eine Diskussion der Interaktion von Geld- und Lohnpolitik möglich ist, sollen hier kurz die Grundlagen eines einfachen, im folgenden verwendeten post-keynesianischen Modells skizziert werden. ${ }^{5}$ In einer geschlossenen Volkswirtschaft ohne staatliche Aktivität wird das Beschäftigungsvolumen (L) bei gegebener Arbeitsproduktivität (y) durch die vom Unternehmenssektor erwartete effektive Nachfrage bestimmt, die die Höhe der Produktion (Y) determiniert:

$$
L=\frac{Y}{y} .
$$

Die effektive Nachfrage setzt sich aus der Nachfrage nach Investitionsgütern (I) und der Nachfrage nach Konsumgütern (C) zusammen:

$$
Y=C+I
$$

5 Übersichten über den post-keynesianischen Ansatz finden sich z.B. bei Arestis (1996) und Lavoie (I992). 
Die Konsumnachfrage ist abhängig von der funktionalen Einkommensverteilung und von der Sparquote aus Löhnen $\left(s_{\mathrm{w}}\right)$ und aus Profiten $\left(s_{\Pi}\right) \cdot{ }^{6}$ Bezeichnet man den Konsum aus Profiten mit $C_{\Pi}$, den Konsum aus Löhnen mit $C_{w}$, die Profitsumme mit $\Pi$, die Lohnsumme mit W und die Profitquote, den Anteil der Profite an der Produktion, mit h, so ergibt sich folgende Konsumfunktion:

$$
C=C_{\Pi}+C_{W}=\left(1-s_{\Pi}\right) \Pi+\left(1-s_{W}\right) W=\left(1-s_{\Pi}\right) h Y+\left(1-s_{W}\right)(1-h) Y .
$$

Bei gegebenen Sparquoten und gegebener Einkommensverteilung sind die privaten Investitionen die wesentliche Determinante der effektiven Nachfrage und daher der Beschäftigung, wie durch Einsetzen der Gleichungen 3 und 2 in Gleichung I und entsprechendes Auflösen deutlich wird:

$$
L=\frac{I\left(r^{e}, i\right)}{s_{\Pi} h+s_{W}(1-h)} \cdot \frac{1}{y} .
$$

Die Investitionen hängen positiv von der erwarteten Profitrate $\left(\mathrm{r}^{\mathrm{e}}\right)$ sowie negativ vom monetären Zins (i) ab. Der Zins ist in einer Geldökonomie für den Einkommensbildungsprozess exogen und wird durch die Zinspolitik der Zentralbank wesentlich beeinflusst, wohingegen sich Geld- und Kreditvolumen endogen durch die teils kreditfinanzierte effektive Nachfrage ergeben. ${ }^{7}$ Die Geldmenge wird also nicht exogen durch die Zentralbank vorgegeben, sondern Geld entsteht in einem zweistufigen Bankensystem dadurch, dass Geschäftsbanken die kreditwürdige Kreditnachfrage des Privatsektors, insbesondere der Unternehmen, zu einem gegebenen Zinssatz befriedigen, hierdurch Buchgeld »aus dem Nichts« schaffen, und die Zentralbank die notwendigen Reserven zu dem von ihr gesetzten Leitzinssatz akkommodiert, sofern die Geschäftsbanken nicht über hinreichend freie Reserven verfügen. Die Zentralbank gibt also den Leitzinssatz vor, und die Marktzinssätze bilden sich nach Laufzeit und Risiko von Krediten durch Aufschläge der Geschäftsbanken auf den Leitzinssatz. Bleiben die Aufschlagsätze bei Ausdehnung des Kreditvolumens langfristig konstant, so kann die Zentralbank mit ihrer auf den kurzfristigen Zins am Geldmarkt zielenden Politik in der Tendenz ebenfalls den langfristigen Zins am Kapitalmarkt steuern (vgl. Smithin 1994: III ff.), der für die Investitionsentscheidungen von zentraler Bedeutung ist.

Sind die technischen Produktionsbedingungen gegeben, so hängt die von den Unternehmen erwartete Profitrate - als zweite Determinante der privaten Investitionen nur noch von der Profitquote und dem Auslastungsgrad der Produktionskapazitäten

6 Hierbei wird unterstellt, dass die Sparquote aus Profiten größer ist als die Sparquote aus Löhnen.

7 Zur Geldmengenendogenität in der post-keynesianischen Theorie vgl. auch Lavoie (I992: I49 ff., I996), Hewitson (I995), Moore (I989) und Smithin (I994: 64 ff.). 
ab. ${ }^{8}$ Letzterer wird von der Entwicklung der effektiven Nachfrage beeinflusst. Die Profitquote (h) wird bei Vernachlässigung von Materialkosten, output-unabhängiger Beschäftigung und Abschreibungen auf den Kapitalstock durch die unternehmerische Preissetzung (p) auf imperfekten Gütermärkten bestimmt, indem die Unternehmen einen Mark-up (m) auf die Lohnstückkosten - als Quotient aus Nominallohnsatz (w) und Arbeitsproduktivität - schlagen:

$$
\begin{gathered}
p=(1+m) \frac{w}{y}, \quad m>0, \\
h=\frac{\Pi}{Y}=\frac{m}{1+m} .
\end{gathered}
$$

Der Mark-up wird insbesondere von der Konkurrenzintensität auf den Gütermärkten bestimmt. ${ }^{9}$ Da der Mark-up die kalkulatorischen oder tatsächlichen Zinszahlungen decken muss, wird der minimal erforderliche Mark-up langfristig durch den Zinssatz beeinflusst, da dieser die langfristig minimal erforderliche Profitrate einer Realinvestition festlegt. In der kurzen Frist haben Zinssatzvariationen jedoch keinen Einfluss auf die Höhe des Mark-up, sondern einen inversen Einfluss auf die Höhe der Investitionen und der Beschäftigung. ${ }^{10}$ Hierbei ist von einer asymmetrischen Wirkung der Geldpolitik auf die Investitionen auszugehen. Durch drastische Zinssatzerhöhungen vermag die Zentralbank zwar letztlich jeden Investitionsboom zu brechen und damit den Beschäftigungsgrad zu reduzieren. Eine noch so deutliche Zinssatzsenkung ist jedoch alleine keineswegs hinreichend, um eine höhere Investitionstätigkeit auszulösen und die Beschäftigung zu erhöhen, wenn sie in Phasen geringer oder sinkender Absatzerwartungen erfolgt und die Unternehmen nicht davon ausgehen, eine über dem Zinssatz liegende Profitrate auch tatsächlich realisieren zu können.

Setzt die Geldpolitik eine dauerhafte Veränderung des Zinssatzes durch, so können hiermit langfristig gleichgerichtete Veränderungen des Mark-up und der Profitquote

8 Die Profitrate (r) als Quotient aus Profitsumme (П) und Kapitalstock (K) lässt sich schreiben als: $\mathrm{r}=\Pi / \mathrm{K}=(\Pi / \mathrm{Y})\left(\mathrm{Y} / \mathrm{Y}^{\mathrm{v}}\right)\left(\mathrm{Y}^{\mathrm{v}} / \mathrm{K}\right)=\mathrm{hu}(\mathrm{I} / \mathrm{v})$, wobei $\mathrm{Y}^{\mathrm{v}}$ für den Vollauslastungsoutput, $\mathrm{u}$ für den Auslastungsgrad und v für den Kapitalkoeffizienten steht.

9 Für eine offene Volkswirtschaft ist die Höhe des Mark-up in den der internationalen Konkurrenz ausgesetzten Sektoren zudem durch das ausländische Preisniveau sowie den Devisenkurs begrenzt.

10 Wie in Hein (1999) gezeigt wird, ist ein inverser Einfluss des Zinssatzes auf die ökonomische Aktivität in post-keynesianischen Wachstums- und Verteilungsmodellen mit variabler Kapazitätsauslastung jedoch nur dann gegeben, wenn die Sparquote aus Zinseinkommen sehr hohe Werte annimmt und die Investitionen deutlich invers auf Zinssatzänderungen reagieren. Empirisch ist Ersteres in entwickelten OECD-Ländern (Deutschland, Frankreich, Großbritannien, USA) gegeben, Letzteres findet man zumindest in einem Teil der untersuchten Länder (Deutschland, Frankreich) (vgl. Hein/Ochsen 2003). 
verbunden sein, da langfristig nur die Produktionsprozesse aufrechterhalten werden, die eine durch den Zinssatz vorgegebene Minimalverzinsung des eingesetzten Kapitals ermöglichen. ${ }^{11}$ Kommt es aufgrund einer Veränderung dieser oder anderer Determinanten des Mark-up zu einer Verschiebung in der funktionalen Einkommensverteilung, so sind die Wirkungen auf Investitionen, Output und Beschäftigungsniveau jedoch nicht eindeutig. Der Gesamteffekt einer Verteilungsänderung auf Produktion, Beschäftigung und Investitionstätigkeit hängt von den Konsumquoten aus Löhnen und aus verteilten Profiten, sowie von den Elastizitäten der Investitionsentscheidungen im Hinblick auf Zinssätze, Stückkostenentwicklung und Auslastungsgrad ab. Hier sind auch in einem post-keynesianischen Modell verschiedene Regime möglich. ${ }^{12}$

\section{Inflation, Beschäftigung und Geldpolitik bei unkoordinierten Lohnverhandlungen}

\section{I Die NAIRU als kurzfristige Inflationsbarriere}

Nachdem soweit die Determinanten von Verteilung und effektiver Nachfrage in dem hier zugrunde gelegten post-keynesianischen Modell skizziert worden sind, kann nun die Interaktion von Geld- und Lohnpolitik analysiert und eine post-keynesianische Interpretation der NAIRU präsentiert werden. In Abbildung I wird hierzu ein Conflicting Claims-Modell von Inflation, Beschäftigung und NAIRU bei konstanter Arbeitsproduktivität dargestellt, das an die neu-keynesianische Arbeit von Carlin/Soskice (I990: I35 ff.) anschließt. ${ }^{13}$ Obwohl Gegenstand der Lohnverhandlungen zwischen Unternehmen und

11 Da in post-keynesianischen Wachstums- und Verteilungsmodellen Profitrate und monetärer Zinssatz sich nicht entsprechen müssen, kann eine dauerhafte Zinssatzerhöhung auch zu Lasten der einbehaltenen Unternehmensgewinne gehen (vgl. Hein 1999). Geht man jedoch von differenzierten Mark-ups aus, die sich innerhalb eines Produktionszweigs bei einheitlichen Preisen und Löhnen aber unterschiedlichen Arbeitsproduktivitäten zwangsläufig ergeben, so kommt es auch bei Nicht-Weitergabe von Zinssatzerhöhungen an die Preise zu einem Anstieg des durchschnittlichen Mark-up, wenn die produktivitätsschwachen Grenzunternehmen ausscheiden, in denen der Mark-up nicht mehr ausreicht, um die nun gestiegenen Zinskosten zu decken. Die empirische Evidenz für die aggregierte Verteilungswirkung einer Zinssatzänderung ist jedoch nicht eindeutig (vgl. Argitis/Pitelis 200I, Hein/Ochsen 2003).

12 Dies ist von Bhaduri/Marglin (1990) für den Zusammenhang von funktionaler Einkommensverteilung und Investitionen sowie von Hein (1999) und Hein/Ochsen (2003) für die Einflüsse des Zinssatzes auf Verteilung und Investitionen gezeigt worden.

13 Es wird hier also mehr an die europäische Variante des Neu-Keynesianismus angeknüpft, in der Verteilungsauseinandersetzungen auf unvollständigen Märkten im Mittelpunkt stehen, als an die US-Variante, die ein stärkeres Gewicht auf Preisrigiditäten auf Gütermärkten und Inflationserwartungen legt. Ähnliche Ansätze wie der hier zugrunde gelegte finden sich auch bei Lavoie (I992: 372 ff.), Layard/Nickell/Jackman (I99I: I2 ff.) und Rowthorn (1977). 
Abbildung I: Beschäftigung, Verteilungskonflikt und Inflation

bei unkoordinierten Lohnverhandlungen

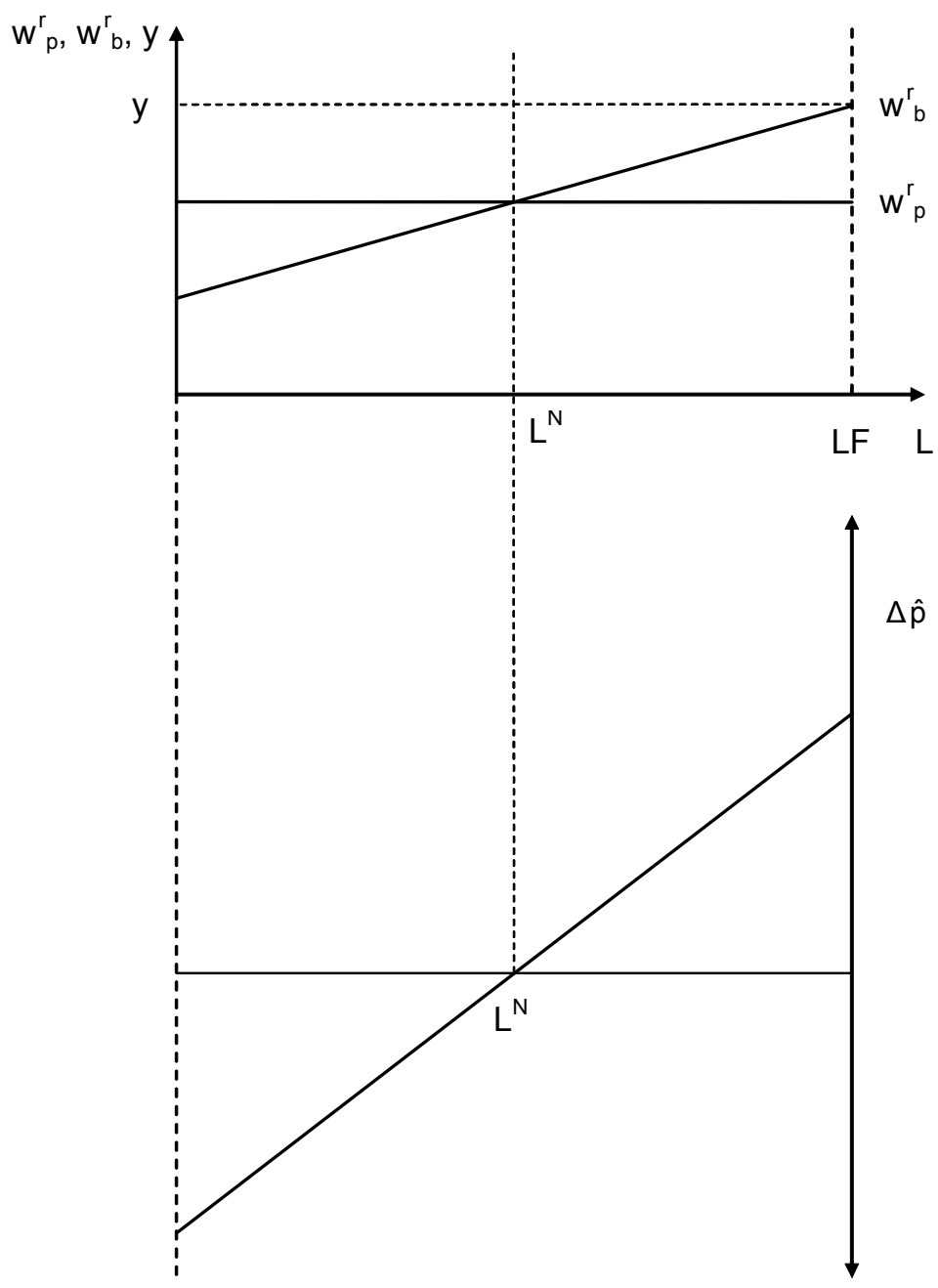

Quelle: Eigene Darstellung

ArbeiterInnen bzw. Gewerkschaften ein Nominallohnsatz ist, wird davon ausgegangen, dass die Gewerkschaften mit ihren Nominallohnsatzforderungen die Durchsetzung eines bestimmten Reallohnsatzes beabsichtigen. Es wird vorerst weiter unterstellt, dass Lohnverhandlungen in den jeweiligen Industriezweigen stattfinden und dass die gewerkschaftlichen Nominallohnforderungen ohne Rücksichten auf mögliche gesamtwirtschaftliche Auswirkungen formuliert werden. Eine gesamtwirtschaftliche Koor- 
dination der Lohnpolitik findet genauso wenig statt wie eine Abstimmung zwischen Lohn- und Geldpolitik. In Abschnitt 4 werden den wirtschaftlichen Ergebnissen bei unkoordinierter Lohnpolitik dann die Wirkungen einer koordinierten Lohnpolitik gegenübergestellt, in der die gesamtwirtschaftlichen Auswirkungen durch die Akteure berücksichtigt werden.

Der von den Gewerkschaften in den Lohnverhandlungen intendierte Reallohnsatz $\left(\mathrm{w}_{\mathrm{b}}^{\mathrm{r}}\right)$, der bei korrekt antizipierter Produktivitätswachstumsrate gleichbedeutend mit einer intendierten Lohnquote ist, hängt positiv von dem durch die effektive Nachfrage bestimmten Beschäftigungsvolumen (L) und, bei als gegeben unterstellter Erwerbsbevölkerung (LF), damit vom Beschäftigungsgrad (L/LF) ab. Bei Vollbeschäftigung würde der Zielreallohnsatz der Arbeitsproduktivität (y) entsprechen. Arbeitslosigkeit hat in diesem Modell also die Funktion, die verteilungs- und wirtschaftspolitischen Beteiligungsansprüche der abhängig Beschäftigten zu zügeln: eine Sichtweise, deren Begründung schon bei Marx (1867) und Kalecki (1943) zu finden ist:

$$
w_{b}^{r}=w_{b}^{r}\left(\frac{L}{L F}, y\right), \quad \quad \operatorname{mit} \frac{\partial w_{b}^{r}}{\partial \frac{L}{L F}}>0, \frac{\partial w_{b}^{r}}{\partial y}>0 .
$$

Dem Zielreallohnsatz der Gewerkschaften steht der durch die Arbeitsproduktivität und durch den Mark-up in der Preissetzungsfunktion der Unternehmen maximal mögliche Reallohnsatz $\left(\mathrm{w}_{\mathrm{p}}^{\mathrm{r}}\right)$ gegenüber. Denn aus Gleichung 5 erhält man:

$$
w_{p}^{r}=\frac{w}{p}=\frac{y}{1+m}
$$

Der Zielreallohnsatz der Gewerkschaften und der durch unternehmerische Preissetzung mögliche Reallohnsatz stimmen nur zufällig überein. Nur wenn das durch die effektive Nachfrage auf den Gütermärkten gegebene Beschäftigungsvolumen zufällig $L^{\mathrm{N}}$ entspricht, sind die Verteilungsansprüche von Gewerkschaften und Unternehmen wechselseitig kompatibel, und es sind keine inflationssteigernde oder -senkende Wirkungen zu erwarten. $L^{\mathrm{N}}$ beschreibt daher die inflationsstabile Beschäftigung. Erwartete und tatsächliche Inflationsrate entsprechen hier einander. Die mit diesem Beschäftigungsvolumen gleichzeitig gegebene Arbeitslosenquote $\left.\left[\left(\mathrm{LF}-\mathrm{L}^{\mathrm{N}}\right) / \mathrm{LF}\right)\right]$ kann daher als Non-Accelerating Inflation Rate of Unemployment (NAIRU) bezeichnet werden. Die NAIRU beschreibt damit ein »Gleichgewicht« zwischen den Verteilungsansprüchen von Arbeit und Kapital. Liegt das durch die effektive Güternachfrage vorgegebene Beschäftigungsvolumen oberhalb von $L^{\mathrm{N}}$, folgen von Periode zu Periode steigende Inflationsraten $(\hat{\mathrm{p}})$ als Ausdruck eines eskalierenden Verteilungskonfliktes. ${ }^{14}$ Liegt dieses Beschäftigungsvolumen unterhalb von $\mathrm{L}^{\mathrm{N}}$, so sind fallende Inflationsraten und letztlich Deflation als Ausdruck einer

14 Die Wachstumsrate der Größe x wird geschrieben als $\hat{x}$. 
erzwungenen Lohnzurückhaltung der Gewerkschaften die Folge. Dieser in Abbildung I dargestellt Zusammenhang ergibt sich aus den Gleichungen 9 bis II:

$$
\begin{gathered}
\hat{w}=\hat{p}^{e}+\hat{y}+\alpha\left(L-L^{N}\right), \quad \alpha>0, \\
\hat{p}=(1 \hat{+} m)+\hat{w}-\hat{y}, \\
\hat{p}-\hat{p}^{e}=(1 \hat{+} m)+\alpha\left(L-L^{N}\right) .
\end{gathered}
$$

Die von den Gewerkschaften durchgesetzten Wachstumsraten des Nominallohns (Gleichung 9) setzen sich zusammen aus der erwarteten Inflationsrate $\left(\hat{\mathrm{p}}^{\mathrm{e}}\right)$, der erwarteten Produktivitätswachstumsrate, für die hier vorerst keine Erwartungsfehler unterstellt werden sollen und die daher der tatsächlichen Produktivitätswachstumsrate entspricht, sowie einer von der Beschäftigungshöhe abhängigen Umverteilungskomponente. Liegt das Beschäftigungsniveau über einem kritischen Niveau $\left(\mathrm{L}^{\mathrm{N}}\right)$, so versuchen die Gewerkschaften ein über das Produktivitätswachstum hinausgehendes Reallohnwachstum durchzusetzen, indem sie ein über den »Verteilungsspielraum« von erwarteter Inflationsrate und Produktivitätswachstum hinausgehendes Nominallohnwachstum durchsetzen. Der Parameter a beschreibt die Intensität mit der Umverteilungsansprüche auf Abweichungen der tatsächlichen Beschäftigung von $\mathrm{L}^{\mathrm{N}}$ reagieren. Liegt das Beschäftigungsniveau unterhalb des kritischen Niveaus, so können Gewerkschaften den Verteilungsspielraum nicht ausschöpfen. Die unternehmerische Preissetzung folgt zeitlich den Lohnverhandlungen, d.h. Unternehmen können zu jeder Zeit auf Veränderungen der nominalen Lohnstückkosten reagieren und diese komplett auf die Preise überwälzen. ${ }^{15}$ Die Wachstumsrate des Preisniveaus kann also wie in Gleichung Io geschrieben werden. Gleichung II zeigt, dass es unter der Voraussetzung eines konstanten Mark-up nur dann zu einer konstanten Inflationsrate kommt $\left(\hat{\mathrm{p}}=\hat{\mathrm{p}}^{\mathrm{e}}\right)$, wenn das Beschäftigungsvolumen nicht von $\mathrm{L}^{\mathrm{N}}$ und die tatsächliche Arbeitslosenquote daher nicht von der NAIRU abweicht. Bildet die tatsächliche Inflationsrate der laufenden Periode die erwartete Inflationsrate für die kommende Periode, so erhält man bei Abweichungen von $\mathrm{L}$ und $\mathrm{L}^{\mathrm{N}}$ kumulative Inflations- bzw. Disinflationsprozesse.

In einem post-keynesianischen Modell, in dem Geld über Gläubiger-SchuldnerVerhältnisse entsteht und von Profit- und Nachfrageerwartungen getriebene Investitionen die wesentliche Determinante der Beschäftigung sind, kann die NAIRU nun nicht, wie in den neu-keynesianischen Modellen, als ein durch endogene (Markt-)Kräfte erzeugtes langfristiges Gleichgewicht interpretiert werden (vgl. Sawyer 200I). Ein solches langfristiges Gleichgewicht setzt voraus, dass sich die tatsächliche Güternachfrage an das

15 Dies ist natürlich eine stark vereinfachende Annahme, wodurch die modellhafte Diskussion erleichtert wird. Allerdings würde die Annahme unvollständiger Überwälzung steigender Lohnstückkosten an den qualitativen Ergebnissen nichts Wesentliches ändern. Lavoie (I992: 39I ff.) analysiert ein solches Modell. 
zur NAIRU gehörende Güterangebot anpasst. Diese Anpassung muss entweder auf die Wirksamkeit von Realkasseneffekten setzen, für die wiederum die Dominanz einer exogen gegebenen Geldmenge unterstellt werden muss, die für die Ökonomie ein Nettovermögen darstellt. Dies ist jedoch bei über Gläubiger-Schuldner-Verhältnisse entstehendem endogenen Geld nicht gegeben. Oder aber eine Anpassung der effektiven Nachfrage an das von der NAIRU implizierte Güterangebot muss über symmetrische Eingriffe und Wirkungen der Geldpolitik abgeleitet werden. Symmetrische Wirkungen der Geldpolitik auf die effektive Nachfrage sind aber für rezessive Phasen mit steigender Arbeitslosigkeit nicht zu erwarten, wie oben bereits begründet wurde.

Sobald die tatsächliche Arbeitslosenquote die NAIRU übersteigt, kann man in einem post-keynesianischen Modell daher vielmehr kumulative Abweichungen der gütermarktbestimmten Arbeitslosenquote von der NAIRU erwarten. Man erhält nämlich bei dann rückläufigen Inflationsraten letztlich Deflation, abnehmende Ertragswertungen der Unternehmen wegen rückläufiger Nachfrage bei Preissenkungserwartungen und eine Aufwertung des Realwertes der nominal fixierten Verbindlichkeiten des Unternehmenssektors (vgl. Fisher 1933). ${ }^{16}$ Abnehmende Profiterwartungen und Aufwertung der Realschuld führen daher zu einem Rückgang der privaten Investitionen und einem weiteren Anstieg der tatsächlichen Arbeitslosenquote, die sich damit immer weiter von der NAIRU entfernt.

Wenn die tatsächliche Arbeitslosenquote die NAIRU unterschreitet, wird ein durch Verteilungskämpfe ausgelöster Prozess steigender Inflationsraten in Gang gesetzt, der seine Grenze in der Geldpolitik der Zentralbank findet, insbesondere bei unabhängigen Zentralbanken mit hoher Präferenz für Preisniveaustabilität. Deren Funktion besteht darin, den Rückfluss des Realwertes eines für die Einleitung eines Produktionsprozesses erforderlichen Kredites bzw. Geldvorschusses und damit die Bereitschaft zu erneuter Kreditvergabe als Voraussetzung für einen kontinuierlichen gesamtwirtschaftlichen Reproduktionsprozess zu garantieren. Eine durch nicht vorhersehbare, kumulative Inflationsprozesse und hiermit verbundene Unsicherheiten ausgelöste Erosion des Geldsystems soll also durch die Zentralbank verhindert werden. Das Instrument der Geldpolitik ist hierbei der Nominalzinssatz am Geldmarkt, mit dem auch der langfristige Zinssatz auf dem Finanzmarkt indirekt und ggf. zeitverzögert beeinflusst wird. Wie in Abbildung 2 gezeigt wird, kann die Zentralbank durch eine Zinssatzerhöhung kurzfristig die Investitionsnachfrage beschränken, so die effektive Nachfrage und damit die Beschäftigung von $\mathrm{L}_{1}$ auf $\mathrm{L}_{2}$ reduzieren.

Fasst man zusammen, so kann die NAIRU in der kurzen Frist durch preisniveaustabilisierende Eingriffe der Geldpolitik stets erzwungen werden, wenn sie von der tatsächlichen

16 Dieser Aufwertung der Realschuld des Defizitsektors (Unternehmen, Staat) muss zwar eine Aufwertung der realen Forderungen des Überschusssektors (private Haushalte) in gleicher Höhe gegenüber gestellt werden. Allerdings geht von dieser Vermögensumverteilung in jedem Fall ein kontraktiver makroökonomischer Effekt aus, solange unterstellt werden kann, dass die Ausgabenquote des Defizitsektors höher ist als die des Überschusssektors. 
Abbildung 2: Kurzfristige Wirkung einer Zinssatzerhöhung

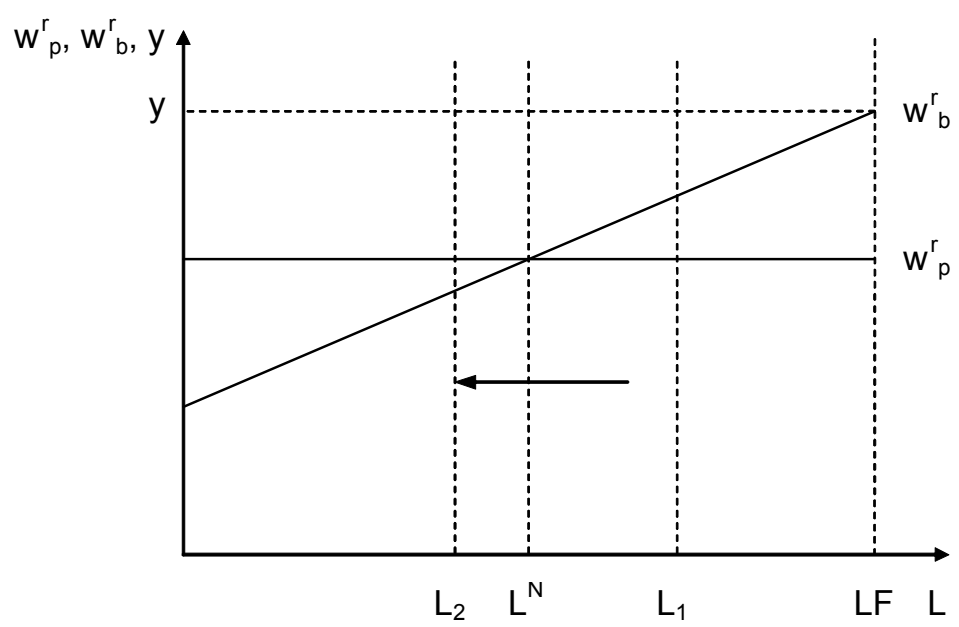

Quelle: Eigene Darstellung

Arbeitslosenquote unterschritten wird. Sie kann jedoch durch die Geldpolitik allein nicht ohne weiteres wieder hergestellt werden, wenn sie von der tatsächlichen Arbeitslosenquote übertroffen wird. In diesem Sinne stellt die NAIRU lediglich eine durch Verteilungskonflikte begründete und geldpolitisch erzwungene Beschäftigungsgrenze oder Inflationsbarriere dar, liefert jedoch keine Erklärung für die gleichgewichtige Arbeitslosigkeit.

\subsection{Die langfristige Endogenität der NAIRU}

Obwohl in einem post-keynesianischen Modell keine Anpassungsmechanismen der gütermarktbestimmten Arbeitslosigkeit an die NAIRU identifiziert werden können, so bestehen doch umgekehrt verschiedene Kanäle, über die sich die NAIRU langfristig an den Entwicklungspfad der tatsächlichen, durch die effektive Güternachfrage determinierten Arbeitslosenquote anpasst. Hierbei sind die von neu-keynesianischen AutorInnen angeführten Argumente für das Hysterese-Phänomen nur ein möglicher Einflusskanal, über den die NAIRU zu einer endogenen Variablen im Hinblick auf die Entwicklung der effektiven Nachfrage wird. Der neu-keynesianische Hysterese-Kanal wirkt dabei über die Verschiebung der gewerkschaftlichen Zielreallohnsatz-Kurve $\left(\mathrm{w}_{\mathrm{b}}^{\mathrm{r}}\right)$ auf die NAIRU: Bei persistent oberhalb der NAIRU liegender Arbeitslosigkeit führen Dequalifizierungs- und Stigmatisierungsprozesse dazu, dass ein Teil der Arbeitslosen keine effektive Konkurrenz auf dem Arbeitsmarkt mehr darstellt und damit die Verteilungsansprüche der abhängig 
Beschäftigten und ihrer Gewerkschaften nicht mehr begrenzt. Die Zielreallohnsatzkurve verschiebt sich für jedes gütermarktbestimmte Beschäftigungsniveau nach oben, und die NAIRU steigt an.

Daneben kann die Entwicklung der effektiven Nachfrage und der tatsächlichen Arbeitslosigkeit aber auch über die Verschiebung des durch die unternehmerische Preissetzung möglichen Reallohnsatzes auf die NAIRU wirken. Erhöht sich die tatsächliche Arbeitslosenquote wegen einer dauerhaften Erhöhung des von der Zentralbank beeinflussten Zinssatzes und wirkt die Zinssatzerhöhung gleichgerichtet auf den Mark-up in der Preissetzung, so verschiebt sich die durch die Preissetzung mögliche Reallohnsatzkurve $\left(\mathrm{w}_{\mathrm{p}}^{\mathrm{r}}\right)$ nach unten und die NAIRU steigt an. Dieser Anstieg wird noch verstärkt, wenn man die Wirkungen des Kapitalstockwachstums auf das Produktivitätswachstum in die Betrachtung einbezieht (vgl. Sawyer 2002). Da eine steigende Arbeitslosigkeit von einem Rückgang der Investitionstätigkeit verursacht wird, rückläufiges Kapitalstockwachstum aber ebenfalls einen Rückgang des Produktivitätswachstums nach sich zieht, ist hiermit ebenfalls die Verschiebung der möglichen Reallohnsatzkurve nach unten verbunden. Wird dieser Rückgang des Produktivitätswachstums - anders als oben modellhaft angenommen - von den Gewerkschaften nicht rational antizipiert und verschiebt sich die Zielreallohnsatzkurve daher nicht gleichzeitig ebenfalls nach unten, so ist mit dem Rückgang des Produktivitätswachstums gleichzeitig ein Anstieg der NAIRU verbunden. Ein weiterer Einflusskanal der effektiven Nachfrage auf die NAIRU ergibt sich für die offene Volkswirtschaft durch den möglichen Effekt eines schwachen inländischen Wachstums auf den Wechselkurs und damit auf den Preis importierter Güter, d.h. auf den Verteilungsanspruch des Auslandes. ${ }^{17}$ Eine geringe inländische Güternachfrage und ein geringes Wachstum kann demnach zu einer Abwertung der inländischen Währung auf internationalen Finanzmärkten führen, weil international agierende VermögensbesitzerInnen aus dem schwachen Wachstum auf geringe zukünftige Erträge inländischer Aktiva schließen und ihre Nachfrage entsprechend einschränken. ${ }^{18}$ Eine Abwertung der inländischen Währung erhöht dann die Importpreise, verschiebt so die mögliche Reallohnsatzkurve nach unten und erhöht die NAIRU.

Der endogene Charakter der NAIRU hat nun zur Folge, dass die langfristigen Verteilungs- und Wachstumseffekte einer Zinssatzerhöhung deren kurzfristige Erfolge bei der Inflationsbekämpfung wieder zunichte machen können. Wirkt eine dauerhafte Zinssatzerhöhung langfristig gleichgerichtet auf die Höhe des Mark-up, so zeigt Abbildung 3, dass sie hiermit den durch die unternehmerische Preissetzung ermöglichten Reallohn von $\mathrm{w}_{\mathrm{p} 1}^{\mathrm{r}}$ auf $\mathrm{w}_{\mathrm{p} 2}^{\mathrm{r}}$ reduziert. Durch den damit verursachten Anstieg der NAIRU - und den entsprechenden Rückgang der inflationsstabilen Beschäftigung von $\mathrm{L}_{1}^{\mathrm{N}}$ auf $\mathrm{L}_{2}^{\mathrm{N}}-$ kann

17 Hein (2002) skizziert ein post-keynesianisches NAIRU-Modell für die offene Volkswirtschaft und zieht es zur Analyse von Lohn- und Geldpolitik im Euroraum heran.

18 Bibow (2002, 2002a) führt die Abwertung des Euros in den Jahren 1999 und 2000 auf eine zu restriktive Geldpolitik der EZB zurück, die eine Wachstumsschwäche im Euro-Raum und einen Rückgang der Nachfrage nach inländischen Aktiva auf internationalen Finanzmärkten wegen der mit der Wachstumsschwäche verbundenen geringen Profiterwartungen ausgelöst hat. 
eine restriktive Geldpolitik wieder das Inflationsproblem hervorrufen, das sie in der kurzen Frist durch Senkung der Beschäftigung von $\mathrm{L}_{1}$ auf $\mathrm{L}_{2}$ (Abbildung 2) erfolgreich bekämpft hat. ${ }^{19}$ Die Berücksichtigung der Effekte einer restriktiven Geldpolitik auf Kapitalstock- und Produktivitätswachstum - sowie auf den Wechselkurs bei Betrachtung einer offenen Volkswirtschaft - verschärft dieses Problem noch. Hierbei wird jeweils unterstellt, dass die Zielreallohnsatzkurve der Gewerkschaften durch die Zinssatzerhöhung und deren Wirkungen nicht verändert wird.

\section{Abbildung 3: Langfristige Wirkung einer Zinssatzerhöhung}

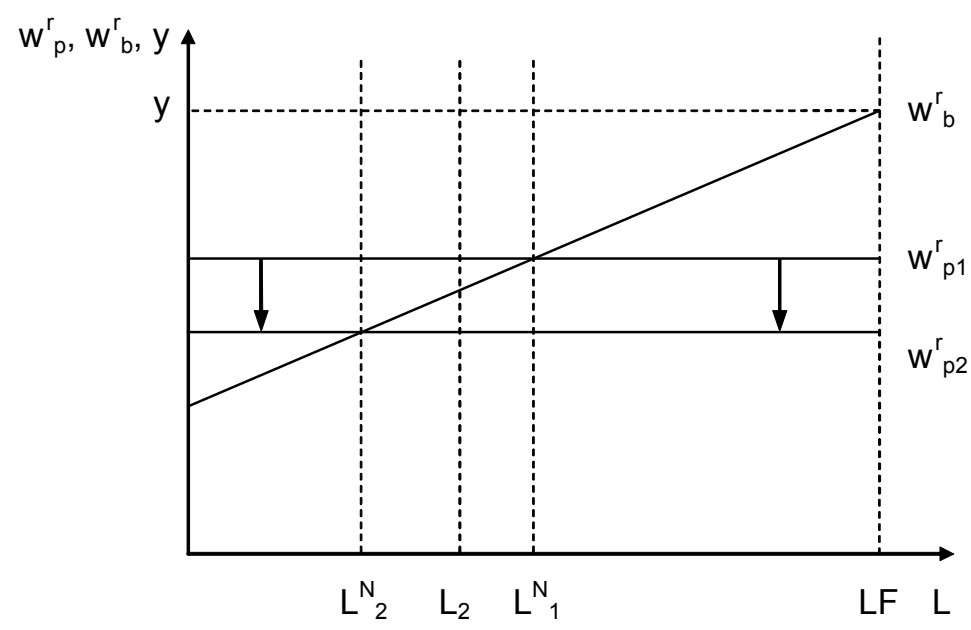

Quelle: Eigene Darstellung

Der Anstieg der Arbeitslosigkeit, der durch die kurzfristigen Wirkungen einer restriktiven Geldpolitik auf Investitionen, effektive Nachfrage und Produktion erzeugt wurde, kann also nicht hinreichend für eine langfristige Stabilisierung der Inflationsrate sein. Eine restriktive Geldpolitik verursacht dann vielmehr das Problem der Stagflation. Eine Reduktion der Inflationsrate kann so eine weitere Restriktion der Geldpolitik und damit einen weiteren Anstieg der NAIRU erfordern, mit allerdings auch wieder nur kurzfristig

19 Hierbei bleiben die Wirkungen unberücksichtigt, die sich aus den gleichgerichteten Effekten höherer Zinssätze auf den Mark-up für die effektive Nachfrage und damit für die tatsächliche Beschäftigung ergeben. Eine Überwälzung der höheren Zinskosten bewirkt letztlich eine Einkommensumverteilung zu Lasten der Löhne. Die Gesamtwirkung von Zinssatzerhöhung und Umverteilung auf Investitionen und effektive Nachfrage sind nun theoretisch unbestimmt (vgl. Hein 1999). Es kann sich sowohl ein erneuter Anstieg von Investitionen und Beschäftigung ergeben als auch ein weiterer Rückgang. Dadurch würden die hier diskutierten Probleme entweder weiter verschärft oder aber entschärft, jedoch nicht beseitigt. 
retardierenden Wirkungen auf die Inflationsrate. Diese Abwärtsspirale aus Inflation, restriktiver Geldpolitik und steigender NAIRU zeigt, dass der Versuch, Inflationsraten durch restriktive Geldpolitik zu stabilisieren, sich nicht nur negativ auf Produktion und Beschäftigung auswirkt, sondern langfristig auch das eigentliche Ziel der Inflationsstabilisierung verfehlen kann.

Aus dem oben skizzierten endogenen Charakter der NAIRU folgt jedoch ebenfalls, dass sich das bei einem gütermarktbestimmten Rückgang der Arbeitslosenquote unter die NAIRU einstellende Inflationspotential nach einer gewissen Zeit endogen wieder abschwächt. Vormals marginalisierte Gruppen werden in den Arbeitsmarkt integriert und entwickeln sich zu einer lohnmoderierenden effektiven Arbeitsangebotskonkurrenz. Hohe Investitionen und starkes Kapitalstockwachstum steigern das Produktivitätswachstum und erhöhen so den Verteilungsspielraum. Hohes inländisches Wachstum kann zu einer Aufwertung der inländischen Währung auf internationalen Finanzmärkten führen, Importpreise senken und so zu einem Rückgang der NAIRU beitragen. Wird der Rückgang der Arbeitslosigkeit unter die NAIRU durch eine expansive Geldpolitik unterstützt, so ermöglichen sinkende Zinssätze einen Rückgang des Mark-up und damit eine Senkung der NAIRU.

Allerdings erfordert eine Senkung der NAIRU durch eine Steigerung der effektiven Güternachfrage, dass die Zentralbank bereit sein muss, steigende Inflationsraten zumindest zeitlich begrenzt zuzulassen. Zudem dürfen die mit steigender Inflation zunehmenden Volatilitäten der Inflationsrate und der damit verbundene Anstieg an Unsicherheit nicht so groß werden, dass die Bereitschaft der VermögensbesitzerInnen und der Geschäftsbanken, Produktionsprozesse zu finanzieren, abnimmt, und sie zur Flucht aus dem betrachteten Währungsraum veranlasst werden. Es stellt sich daher die Frage, ob nicht geeignete Arbeitsmarktinstitutionen und Lohnverhandlungssysteme besser als die Geldpolitik in der Lage sind, einerseits den bei steigender Beschäftigung eskalierenden Verteilungskonflikt zu moderieren und so eine akzelerierende Inflation zu verhindern, andererseits aber auch bei fallender gütermarktbestimmter Beschäftigung das Abgleiten des Systems in Disinflation und Deflation mit den oben skizzierten negativen makroökonomischen Wirkungen zu erschweren.

\section{Der Einfluss der Arbeitsmarktinstitutionen und der Lohnverhandlungssysteme auf Inflation und Beschäftigung}

Der Einfluss von Arbeitsmarktinstitutionen und Lohnverhandlungssystemen auf die makroökonomische Performance ist insbesondere seit der vielzitierten Arbeit von Calmfors/Driffill (I988) in einer Reihe von Untersuchungen thematisiert worden. Hierbei wurden die strategischen Interaktionen von Lohnverhandlungen und Geldpolitik jedoch erst in neueren Studien berücksichtigt. Calmfors/Driffill (I988) stellen bekanntlich die Hypothese auf, dass die Beziehung zwischen dem Zentralisationsgrad der allgemeinen 
Lohnverhandlungen und der Arbeitslosigkeit im zwischenstaatlichen Vergleich humpshaped sei. ${ }^{20}$ Sowohl hochgradig dezentralisierte Systeme, in denen die Lohnfindung auf Betriebs- bzw. Unternehmensebene angesiedelt ist, als auch hochgradig zentralisierte Systeme, in denen die Löhne zentral für die gesamte Ökonomie ausgehandelt werden, weisen ihrer Argumentation zufolge ein geringeres Niveau an Arbeitslosigkeit auf als intermediäre Systeme, in denen die Lohnverhandlungen auf Branchenebene stattfinden. Dieser Zusammenhang wird von Calmfors (1993), ausgehend von einem inversen $\mathrm{Zu}$ sammenhang zwischen Reallohnhöhe und Beschäftigung, durch die unterschiedliche Fähigkeit dieser Systeme zur Reallohnmoderation erklärt. Soskice (I990) zeigt hingegen, dass weniger der Zentralisationsgrad der Tarifverhandlungen einen Einfluss auf die Arbeitslosigkeit hat, sondern dass vielmehr der formelle und informelle Koordinationsgrad der Lohnverhandlungen (z.B. durch Lohnführerschaft bestimmter Gewerkschaften oder Sektoren) sowie der »lokale Lohndruck«, der von der Stärke der Gewerkschaften auf den unteren Ebenen sowie deren Orientierung an kurzfristiger Reallohnmaximierung abhängt, von Bedeutung sind. Hierbei wird dann im Gegensatz zur Hump-shaped-Hypothese ein eindeutig linear-inverser Zusammenhang zwischen Koordinationsgrad und Arbeitslosigkeit sowie ein linear-positiver Zusammenhang zwischen »lokalem Lohndruck" und Unterbeschäftigung abgeleitet.

Traxler (1999) stellt ebenfalls den Koordinationsgrad der Tarifverhandlungen als wesentliche positive Einflussgröße auf die Beschäftigungsentwicklung heraus und macht dabei eine hilfreiche Unterscheidung zwischen der horizontalen und der vertikalen Koordination. Bei funktionierender horizontaler Koordination zwischen den Branchen, z.B. durch Lohnführerschaft, staatliche, inter-verbandliche und intra-verbandliche Koordination oder dreiseitige Abkommen zwischen Tarifparteien und Regierung, gewährleistet erst ein hohes Maß an vertikaler Koordination innerhalb der Branche, z.B. durch einen hohen gewerkschaftlichen Organisationsgrad, einen weiten Geltungsbereich der Kollektivverträge und Friedenspflichten, dass die Ergebnisse der Koordination auch umgesetzt werden. Eine effektive horizontale Koordination der Lohnverhandlungen zwischen den Branchen ermöglicht es, die gesamtwirtschaftlichen Auswirkungen der Lohnbildung, insbesondere im Hinblick auf die Inflationsrate, zu berücksichtigen. Inflationsinkremente zur Absicherung der Branchenverhandlungsergebnisse gegen unvorhergesehene Inflationssteigerungen, die durch Verhandlungsergebnisse in anderen Branchen ausgelöst werden, können damit weitgehend entfallen. Eine effektive vertikale Koordination trägt zur Lösung des Implementierungsproblems der gesamtwirtschaftlich koordinierten Tarifpolitik bei und reduziert die Wahrscheinlichkeit, dass es auf der Unternehmensebene bei hoher Beschäftigung zu positiver Lohndrift oder bei geringer Beschäftigung zum Lohndumping kommt.

20 Vgl. Calmfors (1993), Flanagan (1999) und OECD (1997) für einen umfassenden Überblick über Arbeiten zu Lohnverhandlungssystemen und Arbeitslosigkeit bzw. Inflation sowie zu den konzeptionellen und empirischen Problemen bei der Erfassung der Einflüsse der Arbeitsmarktinstitutionen auf die Makroökonomie. 
Die Bedeutung einer funktionsfähigen horizontalen und vertikalen Koordination der Tarifpolitik für die Internalisierung negativer Lohn-Externalitäten ist dann jüngst auch in einigen Untersuchungen zur strategischen Interaktion zwischen unabhängigen Zentralbanken und Lohnverhandlungen betont worden. ${ }^{21}$ So haben Hall/Franzese (1998) den Einfluss eines Indexes für die Zentralbank-Unabhängigkeit und eines Indexes für den Koordinationsgrad der Lohnverhandlungen auf die jahresdurchschnittliche Inflationsrate sowie auf den durchschnittlichen Misery Index als Summe aus Inflationsrate und Arbeitslosenquote untersucht. Ein höherer Unabhängigkeitsgrad der Zentralbanken ist dabei mit einer geringeren Inflationsrate assoziiert. Vergleicht man dann Länder mit einem ungefähr ähnlichen Unabhängigkeitsgrad der Zentralbanken, so stellt man fest, dass ein höherer Koordinationsgrad der Lohnverhandlungen mit einem geringeren Misery Index verbunden ist. Hieraus folgt, dass in Ökonomien mit einem hohen Koordinationsgrad der Lohnverhandlungen die Inflationsrate durch unabhängige Zentralbanken mit geringeren Beschäftigungsverlusten reduziert werden kann als in Ländern mit einem geringen Koordinationsgrad. In Erweiterung der zitierten Untersuchung hat Franzese (200I) gezeigt, dass die erfolgreiche Internalisierung von Lohnexternalitäten nicht nur vom Koordinationsgrad der Tarifverhandlungen abhängt, sondern auch von der Dominanz des privaten gegenüber dem öffentlichen Sektor und von einem deutlichen Gewicht der Verhandlungen in den Exportindustrien. Die Resultate von Kittel/ Traxler (200I) zeigen zudem, dass die erfolgreiche Antizipation einer restriktiven Geldpolitik entscheidend von einer funktionsfähigen vertikalen Koordination abhängt, die damit das oben bereits angesprochene Implementierungsproblem koordinierter Lohnpolitik zu lösen imstande ist.

Die hier angeführten Arbeiten beschränken sich jedoch darauf, nach institutionellen Bedingungen für Nominallohnmoderation zu suchen, die es ermöglichen, hohe Beschäftigung und geringe Inflation zu kombinieren und damit restriktive Geldpolitiken unabhängiger Zentralbanken zu vermeiden. Es werden weder die makroökonomischen Risiken fallender Nominallöhne oder fallender Lohnstückkosten in Phasen hoher und ansteigender Arbeitslosigkeit noch die langfristigen Einflüsse der effektiven Nachfrage auf den inflationsstabilen Beschäftigungsgrad thematisiert. So kann z. B. die Lohnführerschaft der Exportwirtschaft bei national hochgradig koordinierten Lohnverhandlungen zum Versuch der lohnpolitischen Unterbietung internationaler Konkurrenten führen. Dies kann zwar in kleinen offenen Volkswirtschaften über den Exportkanal durchaus positiv auf Produktion und Beschäftigung wirken. In großen, eher geschlossenen Ökonomien sind bei Verfolgung dieser Strategie jedoch eher die makroökonomisch schädlichen Wirkungen von Disinflation und Deflation zu befürchten.

Die Nichtberücksichtigung dieser beiden Aspekte ist in den hier genannten Studien jedoch nur gerechtfertigt, wenn man davon ausgeht, dass die tatsächliche Arbeitslosenquote sich langfristig immer der von ihr unabhängigen NAIRU annähert. Eine solche

21 Übersichten über Arbeiten zu unabhängigen Zentralbanken und Lohnverhandlungssystemen finden sich bei Franzese (200I, 200Ia). 
Sicht muss jedoch in dem post-keynesianischen Modell aus den oben dargelegten Gründen verworfen werden. Trotzdem können einige Erkenntnisse der hier skizzierten Studien in das post-keynesianische Modell integriert werden. Die Wirkungen einer effektiven Koordination der Tarifverhandlungen, d.h. einer funktionsfähigen horizontalen und vertikalen Koordination im Sinne Traxlers (1999), lassen sich dabei graphisch wie in Abbildung 4 darstellen.

Abbildung 4: Beschäftigung, Verteilungskonflikt und Inflation bei koordinierten Lohnverhandlungen

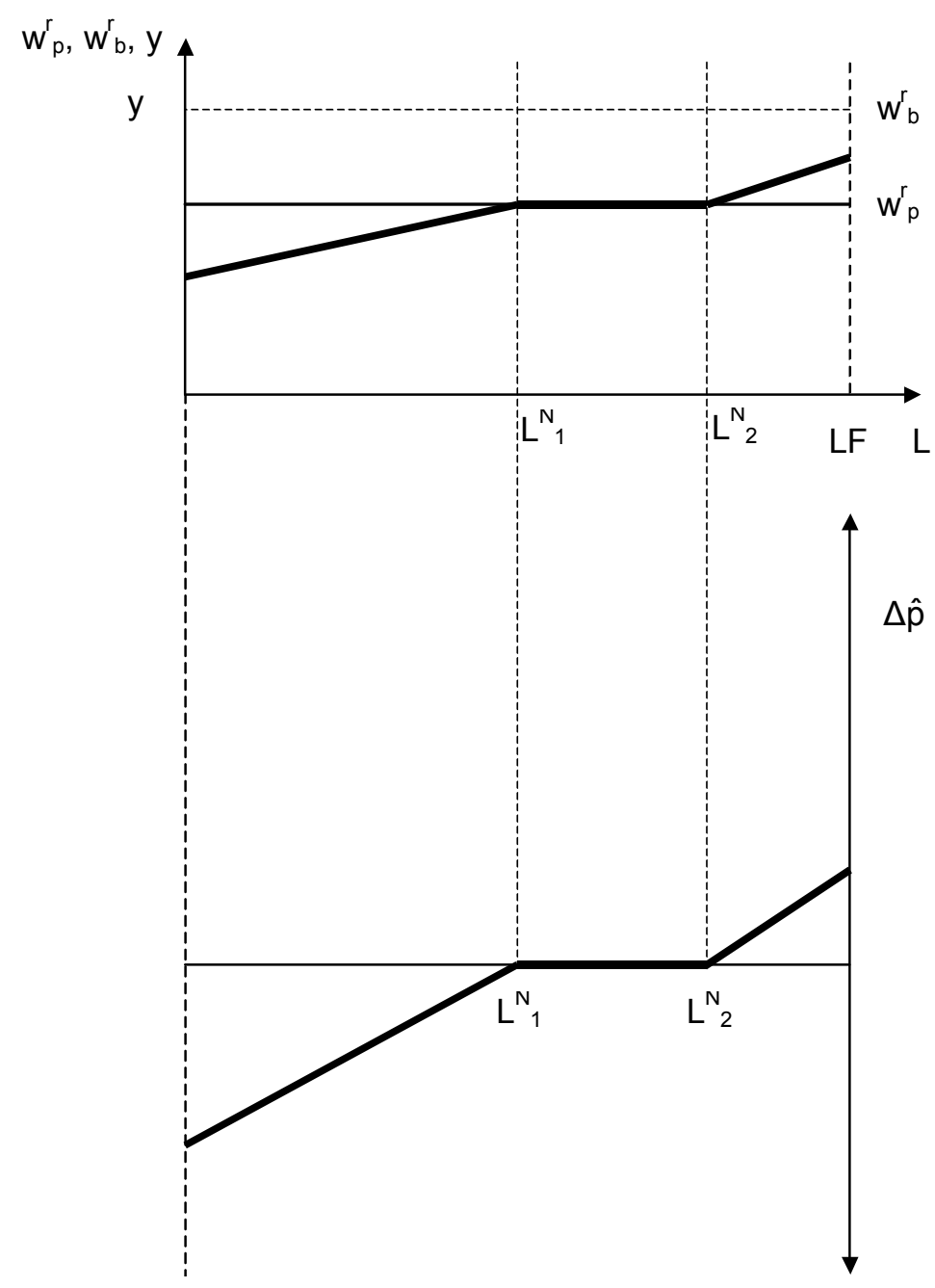

Quelle: Eigene Darstellung 
Liegt eine effektive Koordination der Lohnverhandlungen vor, so verändert sich der Zielreallohnsatz der Gewerkschaften im Vergleich zu unkoordinierten Tarifverhandlungen. Innerhalb eines Bereiches von $\mathrm{L}_{1}^{\mathrm{N}}$ bis $\mathrm{L}_{2}^{\mathrm{N}}$ sind die Tarifparteien in der Lage, den durch den Mark-up in der unternehmerischen Preissetzung gegebenen Verteilungsspielraum anzuerkennen und im Rahmen einer produktivitätsorientierten Lohnpolitik unter Berücksichtigung des Inflationsziels der Zentralbank auszuschöpfen. Fällt die Beschäftigung jedoch unter $\mathrm{L}_{1}^{\mathrm{N}}$, so ist die Verhandlungsmacht der Gewerkschaften soweit geschwächt, dass sie den Verteilungsspielraum nicht mehr auszunutzen vermögen. Steigt die gütermarktbestimmte Beschäftigung über $\mathrm{L}_{2}^{\mathrm{N}}$, so zwingt der Druck der Mitglieder und die Furcht vor positiver Lohndrift die Gewerkschaften dazu, eine Erhöhung des Reallohnsatzes bzw. eine Umverteilung zu Gunsten der Lohneinkommen anzustreben, wodurch ceteris paribus dann die Inflationsrate akzeleriert und ein preisniveaustabilisierender Eingriff der Zentralbank erzwungen wird.

Durch effektive Koordination der Tarifverhandlungen ist eine konstante Inflationsrate also mit sehr vielen, durch den Gütermarkt vorgegebenen Beschäftigungsniveaus verträglich und die NAIRU ist nicht mehr eindeutig. Je höher der Koordinationsgrad, desto höher die Beschäftigung, von der keine inflationsbeschleunigenden Wirkungen ausgehen und desto geringer die minimale NAIRU. Hierdurch eröffnen sich auch für eine ausschließlich an Preisniveaustabilität orientierte Zentralbank erweiterte Möglichkeiten, einen hohen Beschäftigungsstand zu tolerieren. Eine Senkung der NAIRU ist also durch Organisation des Arbeitsmarktes und der Verhandlungspartner möglich und erfordert nicht den Abbau von Rigiditäten durch Dezentralisierung und Fragmentarisierung von Tarifverhandlungen sowie den Abbau von ArbeitnehmerInnenrechten und sozialen Sicherungssystemen. Ein hoher Koordinationsgrad der Tarifverhandlungen verhindert darüber hinaus bei rückläufiger gütermarktbestimmter Beschäftigung, dass es unmittelbar zu Disinflations- und Deflationsprozessen mit negativen Rückwirkungen auf die effektive Nachfrage und auf die Beschäftigungshöhe kommt. Die effektive Koordinierung von Lohnverhandlungen ist daher nicht nur eine der restriktiven Geldpolitik überlegene Methode, wenn es um die Eindämmung von Inflation im ökonomischen Aufschwung geht. Die effektive Koordinierung der Tarifpolitik erlaubt auch, die Ökonomie im Abschwung zu stabilisieren.

\section{Fazit}

Dem neu-keynesianischen langfristigen Gleichgewichtskonzept einer durch Strukturfaktoren des Arbeitsmarktes, der Lohnverhandlungen und der sozialen Sicherungssysteme bestimmten inflationsstabilen Arbeitslosenquote (NAIRU) ist in diesem Beitrag eine postkeynesianische Interpretation der NAIRU gegenübergestellt worden. Die neu-keynesianischen Modelle lassen zwar einen kurzfristigen Einfluss der effektiven Güternachfrage und der Geldpolitik auf die Arbeitslosigkeit und damit eine Abweichung der tatsächlichen 
Arbeitslosenquote von der NAIRU zu, gehen jedoch langfristig davon aus, dass sich die Güternachfrage an das mit der NAIRU kompatible Niveau anpasst. Hierfür muss entweder die Wirksamkeit von Realkasseneffekten gegeben sein oder es müssen symmetrische Reaktionen und Wirkungen der Geldpolitik unterstellt werden. Beide Annahmen sind für eine Geldwirtschaft unhaltbar, in der Geld über Gläubiger-Schuldner-Kontrakte entsteht und in der von Zinssatz und Profiterwartungen gesteuerte Investitionen die wesentliche Determinante von Produktion und Beschäftigung sind.

In einem post-keynesianischen Modell, das diese wesentlichen Eigenschaften einer Geldwirtschaft im Kern berücksichtigt, kann die NAIRU daher nicht als durch Angebotsfaktoren bestimmte Gleichgewichtsarbeitslosigkeit interpretiert werden. Im Rahmen eines post-keynesianischen Modells stellt die NAIRU lediglich eine kurzfristige Beschäftigungsgrenze oder Inflationsbarriere dar, die durch inkompatible und damit inflationsauslösende Verteilungsansprüche gegeben ist und durch die Geldpolitik erzwungen wird. Anpassungstendenzen der tatsächlichen, durch die effektive Güternachfrage bestimmten Arbeitslosenquote an diese NAIRU existieren nicht. Wegen der Dominanz von Real Debt- und Erwartungseffekten sowie der asymmetrischen Wirkung der Geldpolitik ist bei Unterschreitung der NAIRU kurzfristig vielmehr mit kumulativ divergierenden Prozessen von tatsächlicher Arbeitslosenquote und NAIRU zu rechnen.

Langfristig folgt die NAIRU jedoch der tatsächlichen Arbeitslosenquote und wird damit durch die Entwicklung der effektiven Nachfrage bestimmt. Neben dem bereits in einigen neu-keynesianischen Modellen thematisierten Hysterese-Phänomen, nach dem bei dauerhafter Arbeitslosigkeit ein Teil der Arbeitslosen durch Dequalifizierung und Stigmatisierung keine effektive Arbeitsmarktkonkurrenz mehr darstellt, den Zielreallohnsatz der Gewerkschaften nicht mehr beschränkt und dadurch die NAIRU erhöht, sind hier drei weitere Kanäle identifiziert worden. Diese wirken über die Reduktion des Verteilungsspielraums und des durch die Mark-up-Preissetzung möglichen Reallohnsatzes bei geringer effektiver Nachfrage und hoher Arbeitslosigkeit negativ auf die NAIRU. So wirkt sich eine geringe Investitionstätigkeit nicht nur negativ auf die Beschäftigung aus, sondern auch auf das Kapitalstock- und damit auf das Produktivitätswachstum. Geringes inländisches Wachstum kann aufgrund von Erwartungseffekten auf internationalen Finanzmärkten zudem zu einer Abwertung der inländischen Währung führen, die Importe verteuern und damit den inländischen Verteilungsspielraum einschränken. Sind hohe Arbeitslosigkeit und geringe effektive Nachfrage durch steigende Zinssätze verursacht worden, die dann höhere Mark-ups in der Preissetzung nach sich ziehen, so ist hiermit ebenfalls eine Reduktion des Verteilungsspielraums und eine Erhöhung der NAIRU verbunden.

Aus dem zuletzt genannten Zusammenhang folgt auch, dass eine restriktive Geldpolitik nur kurzfristig in der Lage ist, einen kumulativen Inflationsprozess zu brechen, ihn langfristig jedoch wieder anschiebt, so dass sich eine Abwärtsspirale aus Inflation, restriktiver Geldpolitik und steigender NAIRU einstellen kann. Der Versuch, Inflationsraten durch restriktive Geldpolitik zu stabilisieren, wirkt sich daher nicht nur negativ auf Produktion und Beschäftigung aus, sondern kann auch langfristig das eigentliche Ziel, die Inflationsstabilisierung, verfehlen. 
Durch die Einbeziehung von Forschungsergebnissen zum Zusammenhang von Lohnverhandlungssystemen, Geldpolitik und makroökonomischer Performance in das post-keynesianische Modell wurde zuletzt gezeigt, dass effektiv koordinierte Lohnverhandlungssysteme sehr viel besser als restriktive Geldpolitiken in der Lage sind, die Inflationsrate bei hoher Beschäftigung zu stabilisieren. Horizontal und vertikal koordinierte Lohnverhandlungen vermögen, die makroökonomischen Externalitäten der Nominallohnsetzung zu internalisieren. Dadurch kann die NAIRU beträchtlich reduziert werden, wodurch auch eine ausschließlich an Preisniveaustabilisierung orientierte Zentralbank in die Lage versetzt wird, einen hohen Beschäftigungsgrad zu tolerieren. Eine Senkung der NAIRU erfordert daher die Organisation des Arbeitsmarktes sowie der Tarifverhandlungssysteme und nicht die weitere Deregulierung oder die Dezentralisierung von Lohnverhandlungen. Effektiv koordinierte Tarifverhandlungssysteme haben zudem den großen Vorteil, dass sie eine Stabilisierung der nominalen Lohnstückkosten und der Inflationsrate auch bei sinkender Beschäftigung ermöglichen und so ein unmittelbares Abgleiten der Ökonomie in makroökonomisch schädliche Deflationsprozesse verhindern können.

\section{Literatur}

Arestis, P. (1996): Post Keynesian economics: towards coherence, in: Cambridge Journal of Economics, Vol. 20, S. III-I35

Arestis, P., Sawyer, M. (2003): "New consensus«, New Keynesianism and the economics of the »third way", in: Hein, E., Heise, A., Truger, A. (Hg.), Neu-Keynesianismus - der neue wirtschaftspolitische Mainstream?, Marburg, S. 227-244

Argitis, G., Pitelis, C. (200I): Monetary policy and distribution of income: evidence for the United States and the United Kingdom, in: Journal of Post Keynesian Economics, Vol. 23, S. 617-638

Ball, L., Mankiw, N.G. (2002): The NAIRU in theory and practice, in: Journal of Economic Perspectives, Vol. I6(4), S. II5-I36

Bhaduri, A., Marglin, S. (1990): Unemployment and the real wage: the economic basis for contesting political ideologies, in: Cambridge Journal of Economics, Vol. I4, S. $375-$ 393

Bibow, J. (2002): The monetary policies of the European Central Bank and the Euro's (mal)performance: a stability-oriented assessment, in: International Review of Applied Economics, Vol. I6, S. 3I-50

Bibow, J. (2002a): The market versus the ECB, and the euro's plunge, in: Eastern Economic Journal, Vol. 28, S. 45-57

Blanchard, O., Katz, L.F. (1997): What we know and do not know about the natural rate of unemployment, in: Journal of Economic Perspectives, Vol. II (I), S. 5I-72

Calmfors, L. (1993): Centralisation of wage bargaining and macroeconomic performance - a survey, in: OECD Economic Studies, No. 21, S. I6I-I9I

Calmfors, L., Driffill, J. (1988): Centralization of wage bargaining, in: Economic Policy, Vol. 3, S. 13-6I 
Carlin, W., Soskice, D. (1990): Macroeconomics and the Wage Bargain. A Modern Approach to Employment, Inflation and the Exchange Rate, Oxford: Oxford University Press

Clarida, R., Gali, J., Gertler, M. (1999): The science of monetary policy: a New Keynesian perspective, in: Journal of Economic Literature, Vol. 37, S. I66I-I707

Fisher, I. (I933): The debt-deflation theory of great depressions, in: Econometrica, Vol. I, S. $337-357$

Flanagan, R.J. (I999): Macroeconomic performance and collective bargaining: an international perspective, in: Journal of Economic Literature, Vol. 37, S. II5O-II75

Franz, W. (200I): Neues von der NAIRU?, in: Jahrbücher für Nationalökonomie und Statistik, Vol. 22I, S. 256-284

Franzese, R.J. (200I): Institutions and sectoral interactions in monetary policy and wage/ price-bargaining, in: Hall, P.A., Soskice, D. (Hg.), Varieties of Capitalism. Institutional Foundations of Comparative Advantage, Oxford: Oxford University Press, S. IO4-I44

Franzese, R.J. (200Ia): Strategic interaction of monetary policymakers and wage/price bargainers: a review with implications for the European common-currency area, in: Empirica, Vol. 28, S. 457-486

Galbraith, J.K. (1997): Time to ditch the NAIRU, in: Journal of Economic Perspectives, Vol. II (I), S. 93-IO8

Gordon, R.J. (1997): The time-varying NAIRU and its implications for economic policy, in: Journal of Economic Perspectives, Vol. II (I), S. II-32

Hall, P.A., Franzese, R.J. (1998): Mixed signals: central bank independence, coordinated wage bargaining, and European Monetary Union, in: International Organization, Vol. 52, S. 505-535

Hein, E. (1999): Interest rates, income shares and investment in a Kaleckian model, in: Political Economy. Review of Political Economy and Social Sciences, Issue 5, S. 5-22

Hein, E. (2002): Monetary policy and wage bargaining in the EMU: restrictive ECB policies, high unemployment, nominal wage restraint and inflation above the target, in: Banca Nazionale del Lavoro Quarterly Review, Vol. 55, S. 299-337

Hein, E. (2003): Reale und monetäre Analyse: Post-Keynesianismus und Neu-Keynesianismus im Vergleich, in: Hein, E., Heise, A., Truger, A. (Hg.), Neu-Keynesianismus der neue wirtschaftspolitische Mainstream?, Marburg, S. 135-176

Hein, E., Ochsen, C. (2003): Regimes of interest rates, income shares, savings, and investment: a Kaleckian model and empirical estimations for some advanced OECD-economies, in: Metroeconomica, Vol. 54, S. 404-433

Heine, M., Herr, H. (2003): Der Neu-Keynesianismus als neues makroökonomisches Konsensmodell: Eine kritische Würdigung, in: Hein, E., Heise, A., Truger, A. (Hg.), Neu-Keynesianismus - der neue wirtschaftspolitische Mainstream?, Marburg, S. $2 \mathrm{I}-53$

Hewitson, G. (I995): Post-Keynesian monetary theory: some issues, in: Journal of Economic Surveys, Vol. 9, S. 285-310 
Jerger, J. (2003): NAIRU - Theorie, Empirie und Politik, in: Hein, E., Heise, A., Truger, A. (Hg.), Neu-Keynesianismus - der neue wirtschaftspolitische Mainstream?, Marburg, S. 55-84

Kalecki, M. (1943): Political aspects of full employment, in: Kalecki, M., Selected Essays on the Dynamics of the Capitalist Economy, Cambridge: University Press, 197I, S. 138-I45

Kittel, B., Traxler, F. (200I): Lohnverhandlungssysteme und Geldpolitik, in: Wirtschaft und Gesellschaft, Vol. 27, S. II-40

Lavoie, M. (1992): Foundations of Post-Keynesian Economic Analysis, Aldershot: Edward Elgar

Lavoie, M. (1996): Horizontalism, structuralism, liquidity preference and the principle of increasing risk, in: Scottish Journal of Political Economy, Vol. 43, S. 275-300

Layard, R., Nickell, S., Jackman, R. (199I): Unemployment. Macroeconomic Performance and the Labour Market, Oxford: Oxford University Press

Mankiw, N.G. (200I): The inexorable and mysterious tradeoff between inflation and unemployment, in: The Economic Journal, Vol. III, S. C45-C6I

Marx, K. (1867): Das Kapital. Kritik der politischen Ökonomie, Erster Band: Der Produktionsprozeß des Kapitals, Marx-Engels-Werke, Band 23, Berlin 1962

Meyer, L.H. (200I): Does money matter?, in: Federal Reserve Bank of St. Louis Review, Vol. 83 (5), S. I-I5

Moore, B.J. (1989): The endogeneity of credit money, in: Review of Political Economy, Vol. I, S. $65-93$

OECD (1997): Economic performance and the structure of collective bargaining, in: Employment Outlook, July 1997, Paris, S. 63-92

Rowthorn, R.E. (1977): Conflict, inflation and money, in: Cambridge Journal of Economics, Vol. I, S. 215-239

Sawyer, M. (200I): The NAIRU: a critical appraisal, in: Arestis, P., Sawyer, M. (Hg.), Money, Finance and Capitalist Development, Cheltenham: Edward Elgar, S. 220-254

Sawyer, M. (2002): The NAIRU, aggregate demand and investment, in: Metroeconomica, Vol. 53, S. 66-94

Smithin, J. (1994): Controversies in Monetary Economics. Ideas, Issues and Policy, Aldershot: Edward Elgar

Snowdon, B., Vane, H., Wynarczyk, P. (1994): A Modern Guide to Macroeconomics. An Introduction to Competing Schools of Thought, Cheltenham: Edward Elgar

Soskice, D. (1990): Wage determination: the changing role of institutions in advanced industrialized countries, in: Oxford Review of Economic Policy, Vol. 4, S. 36-6I

Staiger, D., Stock, J.H., Watson, M.W. (1997): The NAIRU, unemployment and monetary policy, in: Journal of Economic Perspectives, Vol. I (I), S. 33-49

Stiglitz, J. (1997): Reflections on the natural rate hypothesis, in: Journal of Economic Perspectives, Vol. II, S. 3-10

Traxler, F. (1999): Wage-setting institutions and European Monetary Union, in: Huemer, G., Mesch, M., Traxler, F. (Hg.), The Role of Employer Associations and Labour Unions in the EMU, Aldershot: Ashgate, S. II5-I35 